\title{
La contribución de la geografía de los niños y de las niñas a la enseñanza de la geografía
}

\author{
Stefano Malatesta \\ Università degli Studi Milano Bicocca. Dipartimento di Scienze Umane per la Formazione \\ «Riccardo Massa» \\ stefano.malatesta@unimib.it
}

Jesús Granados Sánchez

Universitat de Girona

jesus.granados@udg.edu

Recibido: junio de 2017

Aceptado: junio de 2017

\begin{abstract}
Resumen
Una de las principales aportaciones de las geografías de los niños y de las niñas a la disciplina geográfica ha sido su inclusión como actores de creación o producción de espacio, ya que lo utilizan y lo viven según sus necesidades y sus criterios, y lo hacen de forma legítima. La escuela debe, por tanto, beneficiarse de este conocimiento y conectarlo con la manera de enseñar la geografía, para que, así, el alumnado comprenda mejor el uso que realizan del espacio. El objetivo del presente artículo es conectar estos dos ámbitos que han permanecido distantes y, para ello, se aporta una propuesta acerca de cómo las geografías de los niños y de las niñas puede incorporarse a la enseñanza de la geografía a partir de los cuatro aspectos principales siguientes: considerar que los niños y las niñas son actores socioespaciales, tener en cuenta el modo cómo se lleva a cabo la apropiación del espacio en la infancia, atender a las primeras representaciones de la experiencia espacial y conocer cómo son los lugares del alumnado que se educa.
\end{abstract}

Palabras clave: geografías de los niños y de las niñas; espacio; lugar; actores socioespaciales; didáctica de la geografía; educación geográfica

\section{Resum. La contribució de la geografia dels nens i les nenes a l'ensenyament de la geografia}

Una de les aportacions principals de les geografies dels nens i de les nenes a la disciplina geogràfica ha estat la inclusió dels infants com a actors de creació o producció d'espai, ja que l'utilitzen i el viuen segons les seves necessitats i els seus criteris, i ho fan de manera legítima. Per tant, l'escola ha de beneficiar-se d'aquest coneixement i connectar-lo amb la manera d'ensenyar geografia, perquè l'ús de l'espai per part de les criatures sigui més ben 
comprès per elles mateixes. L'objectiu del present article és connectar aquests dos àmbits que han caminat de manera paral.lela a partir de l'elaboració d'una proposta sobre com es poden acostar les geografies de la infancia a la didàctica de la geografia, per tal que aquesta última incorpori la recerca i la conceptualització produîda per la primera. Els quatre pilars d'aquest model són: considerar que els nens i les nenes són agents socioespacials, tenir en compte la manera com es du a terme l'apropiació de l'espai a la infancia, atendre les primeres representacions de l'experiència espacial i conèixer de quina manera són els llocs d'infancia de l'alumnat que s'educa.

Paraules clau: geografies dels nens i les nenes; espai; lloc; agents socioespacials; didàctica de la geografia; educació geogràfica

\section{Résumé. La contribution des géographies des enfants à l'éducation en géographie}

L'une des principales contributions des géographies des enfants à la discipline de la géographie est l'inclusion des enfants en tant qu'agents créant de l'espace, car les enfants utilisent et produisent de l'espace aussi bien que les adultes. Nous considérons que les écoles devraient bénéficier de ces connaissances et les inclure dans l'enseignement de la géographie, afin que les enfants puissent mieux comprendre comment ils utilisent l'espace. L'objectif de cet article est de relier les disciplines des géographies des enfants et de l'éducation géographique afin d'enrichir cette dernière. Nous proposons un modèle pour le faire en fonction de quatre idées principales: les enfants doivent être considérés comme des acteurs sociaux produisant de l'espace ; il est important de savoir comment les enfants s'approprient leur espace et leurs lieux ; leur représentation spatiale des lieux et des espaces est essentielle, ainsi que l'étude des lieux des enfants.

Mots-clés: géographies des enfants; espace; lieu; acteurs sociaux; enseignement de la géographie; éducation géographique

\section{Abstract. The contribution of children's geographies to geography education}

One of the contributions made by children's geographies to the field of geography is the inclusion of children as agents that create space, because children use and produce space as well as adults. We understand that schools should benefit from this knowledge and include it in the teaching of geography, so learners can better understand how they use space. The aim of this paper is to connect the fields of children's geographies and geography education in order to enrich the latter. To this end, we propose a model based on four main ideas: children must be considered as social actors that produce space; how children appropriate their space and places is relevant; studying children's spatial representation of places and spaces as well understanding children's places is essential.

Keywords: children's geographies; space; place; social actors; geography teaching; geographic education

\section{Sumario}

1. Introducción 4. El uso educativo de la geografía

2. Marco teórico de las geografías de los niños y de las niñas

3. Principios de la enseñanza del espacio geográfico de los niños y de las niñas

5. Conclusiones

Referencias bibliográficas 


\section{Introducción}

El campo de investigación denominado children's geographies, o 'geografías de los niños y de las niñas', tiene por objeto el estudio de las prácticas, las representaciones y los lugares que definen la experiencia de los niños y de las niñas como actores socioespaciales (Malatesta, 2015). Hay distintas maneras de definir la infancia o qué denominamos niñas y niños. En la Convención internacional sobre los derechos del niño (UNICEF, 1989), se considera que la infancia llega hasta los 18 años. Para nosotros, la infancia relacionada con las geografías de los niños y de las niñas la circunscribimos en la educación primaria, por la importancia del lugar y las representaciones espaciales que se confieren a esta etapa educativa en la mayor parte de los currículos a nivel internacional. Es por ello que, cuando hablemos de infancia a lo largo de este artículo, nos estaremos refiriendo a las edades comprendidas entre los 6 y los 12 años, principalmente. También utilizaremos el concepto de geografías de los niños y de las niñas en plural, para expresar la diversidad de espacios, apropiaciones y experiencias.

El objetivo de este artículo es aportar una propuesta acerca de cómo la geografía de los niños y de las niñas pueden incorporarse a la enseñanza de dicha materia y, así, beneficiarla.

El artículo empieza con una breve exploración teórica del campo de investigación de las geografías de los niños y de las niñas y destaca las principales ideas a tener en cuenta. El siguiente punto desarrolla de forma breve los objetivos de la enseñanza del espacio geográfico. A continuación, se intentan conectar los dos ámbitos anteriores en una propuesta que se estructura a partir de cuatro principios: hay que considerar a los niños y a las niñas como actores socioespaciales, la apropiación del espacio en la infancia tiene sus propias peculiaridades, la enseñanza del espacio geográfico tiene que partir de las primeras representaciones de la experiencia espacial y hay que conocer cómo son los lugares de la infancia del alumnado que se educa.

\section{Marco teórico de las geografías de los niños y de las niñas}

El espacio público y los lugares cotidianos son los ejes temáticos de la reflexión teórica de las geografías de los niños y de las niñas. Su origen se encuentra en dos obras fundamentales: el reconocido libro The Child in the City, donde Colin Ward (1978) hacía una lectura crítica y antiautoritaria del control que el mundo adulto ejercía sobre el espacio urbano y que significó un punto de inflexión en el estudio de la relación entre la infancia y el espacio público. Posteriormente, en el año 2000, las geógrafas Sarah Holloway y Gille Valentine publicaron Children's geograhies: Living, playing, learning and transforming everyday worlds, obra que resultó ser una de las más influyentes en este campo de estudio. Las geografías de los niños y de las niñas se ha considerado estrechamente relacionada con la geografía cultural (Horton y Kraftl, 2006; Baylina Ferré y Prats Ferret, 2010), porque comparte dos 
cuestiones epistemológicas fundamentales de la geografía cultural contemporánea (Yi-Fu Tuan, 1975; Rachel Pain, 2001; Chris Philo, 2000 y 2003) y de otras ciencias sociales: la pluralidad del sujeto geográfico y la extensión de la legitimidad del objeto de estudio. Chris Philo explica que tenemos que ensanchar el espectro de los protagonistas del análisis geográfico e incluir «the geographies of peoples other than white, middle class, middle-aged, ablebodied, sound-minded, heterosexual men» (Philo, 1992: 193). De hecho, los individuos "other than" contribuyen a producir geografías y a transformar lugares a través de las relaciones sociales que establecen en el espacio, a partir de la activación de mecanismos de dotación de significado, representación y apropiación. Gracias a esta reflexión, se ha legitimado la geografía de lo cotidiano como objeto de estudio. De acuerdo con Chris Philo, también «the quite marginal, peripheral, insignificant and even esoteric (...) bits and pieces of human life» (Philo en Horton y Kraftl, 2006: 71), junto a las escalas tradicionales del análisis espacial, como, por ejemplo, la región o la ciudad, también las geografías de los lugares próximos (el aula, la habitación y la casa) y de los pequeños ámbitos de la vida humana pueden convertirse en objeto de investigación geográfica. Aunque, desde esta óptica, todo podría ser objeto de estudio, en la práctica, los expertos en las geografías de los niños y de las niñas (Horton y Kraftl, 2006) han delimitado el campo de investigación en torno a los tres bloques temáticos siguientes: el mecanismo de la reconstrucción de la geografía de lo cotidiano; las características físicas y materiales de los lugares, y la función del cuerpo como el primer instrumento de exploración espacial. La geografía de lo cotidiano pone la lente de observación en lo material y físico del lugar, pero también en lo percibido a través del cuerpo. Por ello interesa estudiar la casa, el aula, la escuela, el parque, la calle, el gimnasio y la plaza o los espacios de juego. Dentro de la materialidad de los lugares, también es importante fijarse en lo que determina la inclusión o la exclusión de los niños y de las niñas, en tanto que son actores sociales en ellos. Dicho de otra manera, es importante que se haya considerado la dimensión, la forma y la distribución de los objetos en el espacio, de tal manera que los niños y las niñas puedan habitarlo.

La metodología de estudio de las geografías de los niños y de las niñas está pensada para centrarse en los lugares. Es lo que Anderson y Jones (2009) han denominado «methodological signifiance of place». Dorothy Moss (2010) expande esta reflexión hablando de la «social complexity of childhood» (2010: 543). Según la autora, se tiene que describir la complejidad que caracteriza la experiencia espacial de los niñas y las niñas a partir de preguntarnos: «¿Cómo ocupan el espacio?», «¿Cómo lo interpretan?», «¿Cómo negocian las reglas y proponen sus roles en cuanto a actores sociales del espacio público?».

\section{Principios de la enseñanza del espacio geográfico}

Uno de los objetivos de la enseñanza de la geografía es que los alumnos aprendan las propiedades espaciales para organizar el conocimiento espacial (la loca- 
lización de los objetos, el límite, la distancia, las relaciones entre objetos, etc.), con el objetivo último de crear sus representaciones espaciales. La enseñanza de la geografía persigue la síntesis del conocimiento espacial a partir de su representación mediante distintas técnicas. Las más utilizadas han sido la cartografía, las narraciones, la iconografía (como, por ejemplo, los mapas mentales), los vídeos y la dramatización.

La didáctica de la geografía cuenta con una larga trayectoria en la enseñanza del espacio. Jesús Granados Sánchez (2010a) identifica cinco aspectos fundamentales sobre dicha disciplina como propuesta de síntesis de otros estudios de diferentes autores y asociaciones geográficas (Gersmehl, 2008; IGU-CGE, 2006; Mérenne-Schoumaker, 2006; Morgan, 2000; Pinchemel, 1982; Reinfried et al., 2007). Los cinco aspectos fundamentales son:

- Conocer dónde están las cosas.

- Entender los lugares, su organización y su funcionamiento.

- Entender las relaciones entre los lugares.

- Comprender la condición humana y la diversidad cultural.

- El desarrollo de la agencia.

Estas ideas centrales se concretan en las siguientes competencias geográficas que propone el autor (Granados Sánchez, 2010b):

- Obtener información del espacio terrestre y de la vida de la humanidad en la Tierra a partir de la observación, de la cartografía y de otras fuentes documentales, así como desarrollar las habilidades espaciales de localización, distribución y representación de la información geográfica, comprendiendo así las dimensiones espaciales de los fenómenos.

- Conocer los lugares y lo que significan para las personas respondiendo cuestiones del tipo: ¿Cómo son los lugares? ¿Cuáles son sus elementos y sus características diferenciadoras? ¿Cómo afectan a la vida de las personas? ¿Cómo actúan las personas en diferentes lugares?

- Desarrollar el sentido de lugar.

- Conocer las relaciones entre los lugares y los territorios, así como entre las personas de territorios diferentes.

- Identificar, entender y explicar cómo son las relaciones entre el espacio y la sociedad, cómo varían en el mundo y cómo van cambiando a lo largo del tiempo. Valorar críticamente las reglas de funcionamiento de los diferentes territorios, de las sociedades humanas en el seno de sus espacios, por ejemplo: el entorno ecológico de las sociedades, la organización social y el peso de las culturas.

- Utilizar el conocimiento y las habilidades geográficas para actuar de forma sostenible y ejercer sus derechos y sus deberes, es decir, resolver problemas y tomar decisiones en la vida diaria, de tal manera que se haga un uso del espacio de manera justa, segura y estética, y ser agentes responsables y consecuentes a todos los niveles, en un mundo global. 


\section{El uso educativo de la geografía de los niños y de las niñas}

La epistemología de las geografías de los niños y de las niñas, como reflexión sobre el objeto de estudio de este campo, aporta cuatro grandes ideas que pueden ayudar a inspirar una enseñanza de la geografía enfocada sobre los lugares y la experiencia infantil, tal y como piden muchos currículos. Estas ideas parten de reconocer a los menores como actores socioespaciales que realizan una apropiación particular de sus espacios de infancia y de los cuales se forman unas representaciones que van ligadas a su experiencia personal. A continuación desarrollamos cada una de estas ideas y proponemos qué uso educativo pueden tener.

\subsection{Los niños y las niñas como actores socioespaciales del hoy}

La primera idea es superar el paradigma de los menores (Holloway y Valentine, 2004) y la noción de la teoría evolutiva de Piaget, que considera a la infancia como una etapa preparatoria para el mundo adulto:

Children, it is commonly assumed, are those subjects who have yet to reach biological and social maturity - quite simply they are younger than adults, and have yet to develop the full range of competencies adult possess [...] This less-than-adult status means that childhood, is a time when children are to be developed, stretched and educated into their future roles, most clearly through the institution of schooling, but also through the family and the wider social and civic life. In the most general sense, then, childhood is understood to be a time of socialization where children learn that it is to be fully human adult beings. (Holloway y Valentine, 2004: 2)

Se trata de considerar a los niños y a las niñas como ciudadanos de pleno derecho, pero con unas peculiaridades sociales concretas que, a la vez, propician un uso determinado del espacio. Alison James y Allan Proud (1990) han introducido esta crítica en su agenda sobre los principios de la infancia y consideran que la condición de dicha etapa humana es un constructo sociocultural a reconstruir, es decir, hay que abandonar la concepción social de infancia como un estadio universal de inmadurez biológica y asumir que los niños y las niñas son actores sociales con plenos derechos. La new social studies of childhood intenta legitimar la infancia como objeto de estudio de la sociología, porque considera que los niños y las niñas son actores sociales tan importantes como el resto de ciudadanos y que constituyen un grupo social más a tener en consideración.

Otra idea que va ligada a esta asunción de la infancia como estadio anterior al mundo adulto es también el carácter futurista de la educación. Desde nuestro planteamiento, queremos criticar la concepción de la escuela como institución de formación de futuros ciudadanos, como la preparación de los niños y de las niñas para el mundo adulto del mañana, ya que creemos que es de vital importancia la educación del hoy, del momento presente vivido por los niños y las niñas, del aprender a reflexionar sobre su experiencia, sus intereses 
y sobre la construcción de sus propios lugares, todo ello con el objetivo de que se perciban a sí mismos como actores socioespaciales. Esta percepción del tiempo como momento vital y del espacio como ámbito de construcción y de convivencia adulta e infantil es la clave de la idea que queremos expresar. Para ello, es necesario superar el «adultocentrismo».

\subsection{Apropiación del espacio}

La apropiación del espacio es individual, social y política. Es individual en tanto que se trata de una experiencia única y propia de cada persona. Es social porque no vivimos solos, sino que lo hacemos en grupo, es decir, en sociedad y a través de las relaciones interpersonales, de ahí que Doreen Massey diga que el espacio está hecho a través de las relaciones. Y es política porque cada vez que ocupamos un espacio es un acto político, ya que expresamos nuestra voluntad sobre qué hacer y cómo utilizar el espacio, lo que, en ocasiones, entra en conflicto con otros intereses o necesidades individuales o del grupo social que también ocupa o utiliza dicho espacio. Así, este deviene esfera política que implica negociación o conflicto.

Si consideramos a los niños y a las niñas como actores sociales que construyen y que se apropian del espacio, entonces tenemos que reconocer que también hacen un uso individual, social y político de aquel, configurando así unos lugares determinados que denominamos lugares de la infancia.

Los lugares de la infancia tienen un gran potencial didáctico, porque nos permiten conocer dónde prefieren estar los niños y las niñas y dónde no; dónde se sienten seguros y dónde no; con quién se relacionan y qué relaciones establecen, y qué relaciones de poder establecen con sus iguales y cómo negocian.

\subsection{La representación de la experiencia espacial}

La geografía de los niños y de las niñas tiende a mostrar las representaciones espaciales que la infancia va creando continuamente como parte de su apropiación y de su creación de los lugares, tanto a nivel individual como colectivo (el grupo clase en la escuela). En cambio, la didáctica del espacio geográfico tiende hacia las representaciones socioespaciales universales. La idea que subyace en nuestra propuesta es ampliar la concepción que tenemos como maestros o educadores sobre las representaciones espaciales de las criaturas, es decir, indagar y valorar más en sus representaciones iniciales y diarias y no obsesionarnos con las propiedades espaciales que deben adquirir y dónde queremos que lleguen en términos de aprendizaje académico. Se trata de empezar de una forma más socioconstructivista, es decir, detectando sus representaciones para después ver cómo ir llegando, a través de la discusión y la reflexión sobre el espacio personal, a una representación social compartida del espacio. Solo así el dominio espacial será completo. A continuación, precisamos los pasos que entendemos se deberían seguir para trabajar la representación espacial teniendo en cuenta la experiencia de los niños y de las niñas en sus lugares de infancia: 
- Indagación continua: indagar en las representaciones espaciales de los niños y de las niñas para informarnos acerca del modo en que ellos construyen los lugares y hacerlo repetidamente, porque la construcción del lugar es continua (en cada momento hacemos representaciones espaciales, tanto personales como sociales $\mathrm{y}$, como resultado, transformamos y creamos los lugares a diario).

- Conciencia, reflexión y conceptualización: reflexionar sobre los conceptos espaciales a partir de los aspectos que aparecen en sus representaciones: hacerles conscientes sobre cómo expresan las cosas e introducir el concepto o la idea geográfica que subyace en ellas y de su significado en el uso espacial.

- La necesidad de compartir: valorar las representaciones espaciales como fruto de la experiencia diaria e introducir la necesidad de compartir las representaciones con los demás. En este momento tiene que aparecer la necesidad de compartir un código.

- La idea de progresión y de expertez: el papel de la didáctica es hacer conscientes a los alumnos de la importancia de adquirir una formación más técnica y especialista, aunque el profesorado tiene que considerar que la competencia se adquiere al final de la etapa educativa, y que mientras tanto se va reflexionando sobre el proceso de aprendizaje (metacognición).

- Exploración de otras maneras de experimentar y de representar el espacio: la representación del espacio va más allá del mapa y de la imagen visual (a partir de la fotografía y del video). Hay que tener en cuenta la experimentación corporal, las distintas percepciones sensoriales diferentes de lo visual, las diversas habilidades físicas, la imaginación, etc. Vivimos el espacio según como lo percibimos, y el cuerpo es muy importante para la manera en que los niños y las niñas experimentan su papel y su sitio en sus lugares de infancia. La representación es un medio en didáctica, pero tiene diferentes formas de concretarse.

\subsection{Los lugares de la infancia}

¿Cuáles son los lugares de la infancia? ¿Cómo podemos utilizarlos desde el punto de vista didáctico?

Los lugares de la infancia raramente aparecen en los libros de geografía y se utilizan poco en la educación primaria. Dentro de la experiencia espacial de los niños y de las niñas, la escuela es el lugar cotidiano más indicado para ser utilizado didácticamente, ya que es donde tienen lugar muchas de las experiencias, conflictivas o no, relacionadas con el espacio; por ejemplo: se puede trabajar en el aula el modo en que ellos utilizan el patio escolar, qué uso se da a cada microespacio, dónde aparece el conflicto, por qué aflora y cómo se puede resolver. Trabajar en los lugares de la infancia permite impartir una educación geográfica consciente: mediante la reflexión sobre hechos y relaciones cotidianas que suceden en sus lugares, los niños y las niñas se dan cuenta de que son actores sociales que tienen un papel determinante en el espacio y que influyen 
decisivamente en él. En cuanto a las implicaciones que esto tiene para el profesorado, hay que destacar el hecho de que permite conocer mejor el modo como ellos y ellas viven, experimentan, comunican, negocian y se enfrentan al conflicto en sus espacios cotidianos.

\section{Conclusiones}

Este artículo ha pretendido construir puentes entre la enseñanza de la geografía y las geografías de los niños y niñas con el objetivo de conseguir que las dos se enriquezcan mutuamente. Las principales ideas que hemos querido transmitir son las siguientes:

- Es necesario crear conciencia en el profesorado y en los niños y niñas de que estos últimos son actores socioespaciales y que tienen que ser tratados como tales.

- Es importante educar para la autonomía de la infancia y hacerla partícipe del proceso de enseñanza y aprendizaje. El protagonismo en educación, por tanto, ha de ser compartido.

- La escuela es parte de una comunidad y de un territorio, y tiene un papel importante en ellos. La escuela, el aula y el patio escolar son lugares de infancia, pero hay muchos otros ámbitos del territorio donde está inserta la comunidad que son importantes para los niños y las niñas. El profesorado tiene que hacer lo posible para llevar la escuela a los lugares de los niños y niñas y traer sus lugares a la escuela para que todos ellos estén interrelacionados.

- Por otra parte, es necesario que el profesorado sea consciente del papel importante que tiene creando lugares de infancia a partir del uso que se realiza del espacio del aula y de la escuela, además del modo como se establecen normas y roles de poder vinculados a esos espacios.

- Y, por último, el profesorado es el encargado de la integración de la percepción personal del espacio en la construcción social de este.

\section{Referencias bibliográficas}

ANDERSON, J. y JONES, K. (2009). «The difference that place makes to methodology: Uncovering the "lived space" of young people's spatial practices». Children's Geographies [en línea], 7 (3), 291-303. $<$ https://doi.org/10.1080/14733280903024456>

Baylina Ferré, M. y Prats Ferret, M. (2010), «The Second International Conference on Geographies of Children, Youth and Families, Barcelona 2009: a report». Children's Geographies [en línea], 8 (4), 437-440. <https://doi.org/10.1080/14733285.2010.515416>

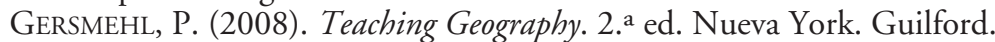

GRANADOS SÁNCHEZ, J. (2010a). «La recerca en l'ensenyament de la geografia per al desenvolupament sostenible». Documents d'Anàlisi Geogràfica, 56 (2), 339-356.

- (2010b). L'Educació per la Sostenibilitat a l'Ensenyament de la Geografia: Un estudi de cas. Tesis doctoral. 
Holloway, S.L. y Valentine, G. (2004). «Children's geographies and the new social studies of childhood». En: Holloway, S. y Valentine, G. (eds.). Children's Geographies: Living, Playing, Learning and Transforming Everyday Worlds. Abington. Routledge, 1-22.

Holloway, S.L. y Valentine, G. (eds.) (2000). Children's Geographies: Living, Playing, Learning and Transforming Everyday Worlds. Abington. Routledge.

HorTON, J. y KrafTL, P. (2006). "What else?: Some more ways of thinking and doing "Children's Geographies"». Children's Geographies [en línea], 4 (1), 69-95. <https://doi.org/10.1080/14733280600577459>

IGU-CGE (International Geographical Union - Commission on Geographical Education) (2006). The International Charter on Geographical Education [en línea]. <http://igu-cge.tamu.edu/cgecharter2005.pdf>.

James, A. y Prout, A. (1990). Constructing and Reconstructing Childhood. Basingstoke. Falmer Press.

Malatesta, S. (2015), Geografia dei bambini: Luoghi, pratiche e rappresentazioni. Milán. Guerini Scientifica.

MÉREnNE-SChOUMAKER, B. (2006). «La enseñanza de la geografía». En: HiernAuX, D. y Lindón, A. (dir.). Tratado de Geografía Humana. México: Editorial Anthropos / Universidad Autónoma Metropolitana-Iztapalapa, 628-643.

Morgan, J. (2002). “"Teaching Geography for a Better World”: The Postmodern Challenge and Geography Education». En: International Research in Geographical and Environmental Education [en línea], 11 (1), 15-29. $<$ https://doi.org/10.1080/10382040208667460>

Moss, D. (2010). «Memory, space and time: Researching children's lives». Childhood [en línea], 17 (4), 530-544. <https://doi.org/10.1177/0907568209345611>

PAIN, R. (2001). Introducing social geographies. Londres. Hodder Education Publisher.

PHILO, C. (1992). «Neglected rural geographies: A review». Journal of Rural Studies [en línea], 8, 193-207. <https://doi.org/10.1016/0743-0167(92)90077-J>

- (2000). "The Corner-stones of my world: Editorial introduction to the Special Issue on Spaces of Childhood». Childhood, 7 (3), 243-256. <https://doi.org/10.1177/0907568200007003001>

- (2003). "To go back up the Side Hill: Memories, imaginations and reveries of childhood». Children's Geographies [en línea], 1 (1), 7-23. <https://doi.org/10.1080/14733280302188>

PINCHEMEL, P. (1982). «De l'enseignement géographique à l'éducation géographique». Historiens-Géographes, 289, 779-783.

Reinfried, S.; Schleicher, Y. y Rempfler, A. (2007). «Geographical views on Education for sustainable development». Proceedings of the Lucerne-Symposium Switzerland, del 29 al 31 de julio. En: Geographiedidaktische forschungen IGU-UGI. Lucerna (Suiza).

TuAn, Y.F. (1975). «Place: an experiential perspective». Geographical Review [en línea], 65 (2), 151-165. $<$ https://doi.org/10.2307/213970>

UNICEF (1989). Convención internacional sobre los derechos del niño [en línea]. $<$ https://www.unicef.es/causas/derechos-ninos/convencion-derechos-ninos>.

Ward, C. (1978). The Child in the City. Londres. The Architectural Press. 